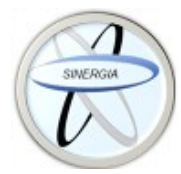

Revista Médica Sinergia

Vol. 6, Núm. 8, agosto 2021, $\underline{\mathrm{e} 696}$

\title{
Actualización de lumbalgia en atención primaria
} Updated of low back pain management in primary care

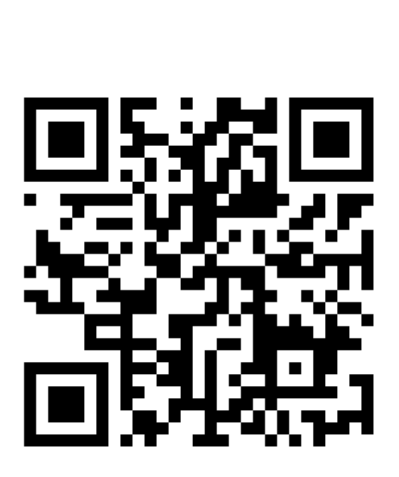

Recibido $16 / 06 / 2021$

\author{
${ }^{1}$ Dra. Michelle Dada Santos \\ Hospital Calderón Guardia, San José, Costa Rica \\ (i) https://orcid.org/0000-0003-3496-1128 \\ ${ }^{2} \mathrm{Dr}$. Andrés Zarnowski Gutiérrez \\ Investigador independiente, San José, Costa Rica \\ (1D https://orcid.org/0000-0001-9356-0100 \\ ${ }^{3}$ Dra. Andrea Salazar Santizo \\ Investigadora independiente, San José, Costa Rica \\ (1D) https://orcid.org/0000-0003-1036-0934
}

Corregido

27/06/2021

Aceptado

20/07/2021

\section{RESUMEN}

La lumbalgia es un síntoma muy frecuente en la atención primaria, tanto en consulta externa como en el servicio de emergencias. Puede tener muchas causas, desde leves hasta severas. Sin embargo, en el $90 \%$ de los casos no se logrará encontrar una causa específica del dolor, esto se llamará lumbalgia mecánica.

Para un adecuado diagnóstico de la lumbalgia, se requiere de una anamnesis y un examen físico exhaustivo, esto nos ayudará a encontrar la etiología, descartar banderas rojas y dar un adecuado manejo.

El manejo de la lumbalgia mecánica se centrará en mejorar el dolor y la calidad de vida del paciente, también en disminuir los gastos en los servicios de salud. Para lograr esto el paciente se debe de proponer realizar ejercicios de estiramiento y fortalecimiento en el hogar, tomar el medicamento indicado y mantener una adecuada dieta y rutina de ejercicios. La labor del médico es tratar de impedir que la lumbalgia aguda evolucione a crónica, esto se logra con actividad física, evitando el reposo en cama, no suspendiendo labores, permaneciendo activo, descartando "banderas amarillas" y sobre todo no realizar estudios médicos innecesarios.

PALABRAS CLAVE: lumbalgia; dolor agudo; dolor crónico; ausencia por incapacidad; atención primaria.

\section{ABSTRACT}

Low back pain is a very frequent symptom in primary care, both in external consultation and in the emergency service. It can have many causes, from mild to severe. However, in $90 \%$ of 
cases it will not be possible to find a specific cause of the pain, this will be called mechanical low back pain.

For an adequate diagnosis of low back pain, an anamnesis and a thorough physical examination are required, this will help us to find the etiology, rule out Red Flags and give proper management.

The management of mechanical low back pain will focus on improving the pain and quality of life of the patient, as well as reducing expenses in health services. To achieve this, the patient should propose stretching and strengthening exercises at home, taking the indicated medication and maintaining an adequate diet and exercise routine. The job of the doctor is to try to prevent acute low back pain from evolving to chronic, this is achieved with physical activity, avoiding bed rest, not suspending work, remaining active, discarding Yellow Flags and above all not conducting unnecessary medical studies.

KEYWORDS: low back pain; acute pain; chronic pain; sick leave; primary health care.

\footnotetext{
${ }^{1}$ Médica ortopedista y traumatóloga - subespecialista en cirugía artroscópica, graduada de la Universidad de Costa Rica (UCR) - Universidad de Barcelona. Cód. MED6902. Correo: dramdadasantos@gmail.com

${ }^{2}$ Médico general, graduado de la Universidad Latina de Costa Rica (U. Latina). Cód. MED16849. Correo: andreszarnowski@gmail.com

${ }^{3}$ Médica general, graduada de la Universidad de Ciencias Médicas (UCIMED). Cód. MED17005. Correo: andre.salazar14@hotmail.com
}

\section{INTRODUCCIÓN}

La lumbalgia es un síntoma muy conocido para todos los médicos de atención primaria, también es una de las consultas más frecuentes ya que es uno de los padecimientos más frecuentes en la vida adulta. Este padecimiento también se encuentra en niños, adolescentes, adultos y adultos mayores, ya no es una patología del adulto como se creía con anterioridad.

La lumbalgia puede tener muchas causas, ya que esto es un síntoma y no una patología como tal. Por definición es un dolor que va desde la última costilla hasta el pliegue de los glúteos. Por esta razón es tan importante la búsqueda del verdadero causante del síntoma. En ocasiones se logrará encontrar la causa específica y en otras ocasiones, posterior a una adecuada historia clínica y examen físico, no se logra encontrar la noxa por lo que se llamará lumbalgia mecánica, esta última es la más frecuente y constituye el $90 \%$ de los casos.

El objetivo del artículo es la actualización, del médico general, en lumbalgia para beneficiar tanto al paciente con un mejor pronóstico de la enfermedad, como al gasto en salud pública que tanto se está viendo afectado por este padecimiento. Recordemos que la lumbalgia es la principal causa de incapacidad en personas de 14-50 años, por eso es tan importante manejar adecuadamente una lumbalgia para ayudar al paciente con no disminuir su pobreza al no trabajar, tener una mejor calidad de vida al ser funcional tanto social como individualmente y el gasto en salud en no realizar tantas incapacidades innecesarias, referencias al servicio de emergencias 0 especialistas y el uso de estudios costosos los cuales no traerán ningún beneficio a la lumbalgia.

\section{MÉTODO}

Se seleccionan los artículos para la revisión presentada con ayuda de la biblioteca de la UCIMED, búsquedas UpToDate, MedLine, Google Scholar. Entre los artículos seleccionados se encuentran tanto en inglés como en español con fechas de publicación 
desde 2016 hasta el 2021. Todos los artículos fueron seleccionados para abordar el tema expuesto tanto de Lumbalgia Inespecífica como los diferentes tipos que existen, esto para darle al lector una mejor idea sobre los tipos y como se maneja cada uno, siempre con el objetivo de que el médico se actualice en el manejo de la lumbalgia y se unifiquen los mismos en los diferentes centros de atención.

\section{DEFINICIÓN}

- Lumbalgia: dolor, tensión muscular o entumecimiento que se localiza de manera dorsal desde el borde inferior de la última costilla hasta el pliegue de los glúteos, con o sin irradiación a miembro inferior (15).

Existen varias definiciones que agregan o no la irradiación a miembro inferior, sin embargo, se cree que esta es la más apropiada ya que le da una visión global sobre la lumbalgia y le permite al médico tratante saber que la lumbalgia puede irradiar sin orientarnos definitivamente a una radiculopatía.

- Lumbalgia mecánica: Cuando no hay una causa clara de la lumbalgia aguda se considera como no especifica, para este grupo, que es la mayoría, no requieren de estudios de imágenes en la primera visita y no requieren de suspender los labores (5).

- Lumbalgia especifica: como su nombre lo indica, tiene un factor específico que está causando el dolor lumbar puede deberse a: tumor, fractura, alteración anatómica de la columna congénita o adquirida, trauma, hernia disco, etc. (ver tabla 1) y requiere de estudios por imágenes y su manejo especifico por especialista (5).

El tiempo de evolución es importante ya que según su clasificación se deberán tomar medidas diferentes en su manejo.
- Lumbalgia aguda: dolor de novo en la zona lumbar que dura menos de 6 semanas con o sin irradiación a miembros inferiores o dolor en zona lumbar con más de 6 meses sin dolor previo al evento por no más de 6 semanas (5).

- Lumbalgia subaguda: dolor lumbar mayor a 6 semanas, pero menor a 12 semanas $(5,12)$.

- Lumbalgia crónica: dolor lumbar con o sin irradiación a miembro inferior con una duración mayor a 3-6meses (4).

\section{EPIDEMIOLOGIA}

La lumbalgia se ha propuesto como la mayor causante de discapacidad a nivel mundial actualmente. Es un síntoma frecuente que ocurre en pacientes de todas las edades, sin embargo, es poco frecuente en menores de 10 años (1). Se propone que el $40 \%$ de las personas de 9-18 años presentarán lumbalgia (1) y hasta el $84 \%$ de los adultos van a presentar lumbalgia una vez en la vida (6), con un promedio de 1 año de duración (1).

Se puede observar un mayor número de mujeres en los casos de consultas por lumbalgia $(1,15)$. A este número se le agregan las mujeres que presentan lumbalgia durante el embarazo, lo cual es tan frecuente como un $67 \%$ (7).

El $85-90 \%$ de las lumbalgias serán inespecíficas y autolimitadas las cuales resolverán en menos de 6 semanas $(6,10)$, sin embargo, de un $24-87 \%$ tendrán otro episodio de lumbalgia en el siguiente año $(2,15)$.

Solo un $5-10 \%$ de las lumbalgias agudas van a convertirse en crónicas, y el deber del médico es evitar esta evolución (9).

La lumbalgia afecta mayoritariamente a personas trabajadoras, más de maquinaria pesada que produzca vibración y de campo (15). 
Hay que recordar que, si el trabajador se ausenta a laborar por una incapacidad no justificada, no solo recibe menos dinero, sino también que produce menos para la sociedad y se gastan recursos de la salud, esto crea un círculo de pobreza (1). Es importante recalcar que el ausentismo al trabajo, por incapacidades, llega a causar un retiro del trabajo anticipado y esto le genera hasta un $87 \%$ menos de riqueza al paciente, comparándolo con una persona que nunca dejó de trabajar (13).

\section{FACTORES DE RIESGO}

Antes de hablar de la etiología, se le quiere brindar al lector una lista de factores de riesgo que le darán una idea de posibles desencadenantes a la hora de realizar la historia clínica.

El fumado, obesidad, edad avanzada, sexo femenino, situaciones de estrés tanto en el hogar como en el trabajo, trabajos con maquinaria pesada o de campo con levantamiento pesado, sedentarismo, poco cuido de la salud, abuso de sustancias, ansiedad y depresión $(10,15)$.

Estos son factores de riesgo para presentar una lumbalgia y para empeorar su cuadro, también algunos de estos factores se comparten con las banderas amarillas de las lumbalgias, las cuales se revisarán más adelante. Los factores de riesgo ayudan al pronóstico de la lumbalgia.

\section{ETIOLOGÍA}

Cuando se busca la causa de la lumbalgia, hay que recordar que esto es un síntoma y no una patología, por lo que hay que sospechar de muchas razones. Si bien es cierto, un dolor de espalda puede ser causado por algo especifico, en el $85 \%$ de los casos no se logra encontrar la causa, a esto le llamamos lumbalgia inespecífica.
El saber que en la mayoría de los casos la lumbalgia será benigna, es importante ya que nos va a permitir realizar una mejor anamnesis y un examen físico completo para descartar las causas que verdaderamente son importantes de descartar.

Primeramente, en varios estudios se evidencia como se diferencian las posibles causantes, ya sea en la columna o fuera de la columna. Hay que recordar que la columna vertebral va a poseer discos intervertebrales, ligamentos, músculos, fascias y nervios, cualquiera de estas estructuras podría producir dolor al lesionarse o traumatizarse, puede ser de manera inocua, autolimitada o seria para el paciente y las estructuras dañadas. También hay que recordar, que fuera de la columna lumbar, pero adyacente a ella, encontramos la cavidad abdominal en donde se encuentran los diferentes órganos que ya todos conocen.

Estos órganos pueden afectarse y producir una alteración localizada en el órgano o bien que afecte otros de manera contigua y produzca que el dolor se irradie de manera dorsal, y se localice en la zona lumbar. Asimismo, existen otras causantes que pueden dar dolor lumbar por ejemplo la piel, si hay una infección o bien una alteración de esta, se puede presentar como una lumbalgia y no necesariamente se va a requerir de estudios complementarios.

En el cuadro 1 se podrán revisar diferentes etiologías extra espinales de la lumbalgia y en el cuadro 2 se encuentran las diferentes causas de lumbalgia mecánica por alteraciones estructurales.

Una vez conocidas las diferentes etiologías que pueden causar lumbalgia se podrá abordar de tal manera que descarte las severas y peligrosas. Las más importantes de descartar son a las que le llaman las banderas rojas, estas podrán ser observadas y estudiadas en la tabla 1. 
Cuadro 1. Causas de lumbalgia fuera de la columna lumbar

1. Abdominales y viscerales: colelitiasis, colecistitis, pancreatitis, tumores, apendicitis.

2. Patología vascular: aneurisma de aorta abdominal.

3. Patologías urológicas: nefrolitiasis, cólico renal, tumor renal, absceso perirrenal, prostatitis.

4. Etiología ginecológica: endometriosis, tumores pélvicos, embarazos.

5. Patologías neurológicas: polineuropatía herpes zoster.

6. Patologías psiquiátricas y psicosomáticas.

Fuente. Casser et al., 2016, "box 3" sección.

\section{Cuadro 2. Causas más frecuentes de lumbalgia mecánica}

\section{Alteraciones estructurales}

- Lumbalgia por sobrecarga funcional y postural

- Hipotonía muscular abdominal

- Hipertonía muscular posterior

- Sobrecargas articulares y discales

- Deportivas

- Sedentarismo

- Embarazo

- Escoliosis

- Hiperlordosis

- Patología discal

- Artrosis interapofisarias posteriores

- Dismetrías pélvicas

- Espondilólisis

- Espondilolistesis

\section{Traumatismo}

- Distensión lumbar

- Fracturas de compresión: de cuerpos vertebrales y de apófisis transversas

- Subluxación de la articulación vertebral

- Espondilolistesis: fractura traumática del istmo

Fuente. Movasat Hajkhan et al., 2017, "Tabla2" sección
Tabla 1. Banderas de alarma para la lumbalgia

\section{Banderas amarillas}

1. Depresión o estrés ocupacional,

2. Proceso cognitivo hacia el dolor (catastrófico o sin esperanza)

3. Comportamiento evitativo por miedo al dolor

4. Problemas psiquiátricos

5. Tendencia a la somatización

6. Ganancias secundarias

7. Otros procesos patológicos crónicos coexistentes

8. Insatisfacción laboral

9. Malos comportamientos hacia la dieta y ejercicio

\begin{tabular}{|c|c|}
\hline \multicolumn{2}{|r|}{ Banderas rojas } \\
\hline Cáncer & $\begin{array}{l}\text { 1. Mayores de } 50-60 \text { años } \\
\text { 2. Historia de tumor previo, nos } \\
\text { hace pensar en metástasis } \\
\text { 3. Síntomas B: Fiebre mayor a } \\
38^{\circ} \mathrm{C} \text {, sudoración nocturna, } \\
\text { pérdida de peso no deseado } \\
\text { (>10\% del peso corporal en } \\
6 \text { meses) } \\
\text { 4. Dolor que empeore en } \\
\text { posición supina } \\
\text { 5olor lumbar intenso por la } \\
\text { noche }\end{array}$ \\
\hline $\begin{array}{l}\text { Infección } \\
\text { vertebral }\end{array}$ & $\begin{array}{l}\text { 1. Síntomas B } \\
\text { 2. Dolor intenso en la noche o } \\
\text { que no mejora en reposo } \\
\text { 3. Historia de infección } \\
\text { bacteriana previa } \\
\text { 4. Procedimientos espinales } \\
\text { invasivos previos } \\
\text { 5. Drogas intravenosas } \\
\text { 6. Inmunosupresión }\end{array}$ \\
\hline $\begin{array}{l}\text { Cauda } \\
\text { equina }\end{array}$ & $\begin{array}{l}\text { 1. Incontinencia fecal, } \\
\text { incontinencia urinaria, o } \\
\text { retenciones urinarias } \\
\text { 2. Hiperestesia } \\
\text { perianal/perineal } \\
\text { 3. Debilidad de neurona motora } \\
\text { inferior persistente o } \\
\text { progresiva }\end{array}$ \\
\hline $\begin{array}{l}\text { Dolor } \\
\text { radicular }\end{array}$ & $\begin{array}{l}\text { Dolor lumbar con dolor de } \\
\text { miembro inferior que afecta la } \\
\text { raíz de L4, L5 o S1, por esta } \\
\text { razón se verá afectado todo el } \\
\text { dermatomo con debilidad } 3 / 5 \text {, } \\
\text { parestesias y perdidas de los } \\
\text { reflejos osteotendinosos }\end{array}$ \\
\hline
\end{tabular}




\begin{tabular}{|c|c|}
\hline \multicolumn{2}{|c|}{$\begin{array}{l}\text { Tabla 1. Banderas de alarma para la } \\
\text { lumbalgia }\end{array}$} \\
\hline $\begin{array}{l}\text { Estenosis } \\
\text { espinal }\end{array}$ & $\begin{array}{l}\text { 1. Mas frecuentes en adultos } \\
\text { mayores } \\
\text { 2. Puede dar parestesias, dolor } \\
\text { y debilidad en glúteo, muslo } \\
\text { o pierna } \\
\text { 3. Produce pseudoclaudicación } \\
\text { que mejora cuando se inclina } \\
\text { hacia delante } \\
\text { 4. Puede producir muerte de la } \\
\text { raíz nerviosa, mejora el } \\
\text { dolor, pero pierde totalidad } \\
\text { de la función }\end{array}$ \\
\hline & $\begin{array}{l}\text { 1. Traumatismo severo: } \\
\text { accidente de tránsito, caída } \\
\text { desde gran altura, accidente } \\
\text { de deporte } \\
\text { 2. Trauma menor: ya sea por } \\
\text { levantar peso, toser o } \\
\text { estornudar. Sospechar de } \\
\text { osteoporosis en personas } \\
\text { mayores } \\
\text { 3. Uso crónico de esteroides } \\
\text { sistémicos }\end{array}$ \\
\hline $\begin{array}{l}\text { Tiempo } \\
\text { evolución }\end{array}$ & $\begin{array}{l}\text { Un tiempo de evolución mayor a } \\
6 \text { semanas, con apego al } \\
\text { tratamiento médico y } \\
\text { rehabilitación en el hogar, sin } \\
\text { mejoría alguna del dolor }\end{array}$ \\
\hline \multicolumn{2}{|c|}{$\begin{array}{l}\text { Fuente. }{ }^{1} \text { Pérez Delgado FJ, Gil Sánchez C, } \\
\text { Izquierdo Gomar AB. Lumbalgia resistente a } \\
\text { tratamiento. Med gen fam. 2016;5(2):68-70. 2Urits } \\
\text { I, Burshtein A, Sharma M, Testa L, Gold PA, } \\
\text { Orhurhu V, et al. Low back pain, a comprehensive } \\
\text { review: Pathophysiology, diagnosis, and treatment. } \\
\text { Curr Pain Headache Rep. 2019;23(3):23 }\end{array}$} \\
\hline
\end{tabular}

Es importante recordar, como se mencionó anteriormente, que el $85-90 \%$ de las lumbalgias son benignas y que estas serán autolimitadas, lo que significa que dejarán de existir por sí solas en menos de 6 semanas. $(6,10)$. De las lumbalgias agudas la más frecuente es causada por una contractura muscular de los músculos dorsales (2). Menos del $1 \%$ tendrá una patología sistémica grave tipo cáncer o infección, menos del $10 \%$ serán etiologías como fractura, radiculopatía o estenosis espinal (10).
La lumbalgia mecánica, es la más frecuente de las causas de lumbalgia, se puede considerar como una lesión más de las causadas por la actividad física (o movimientos) de la vida cotidiana, de la actividad laboral o profesional y del tiempo libre (ejercicio físico/deporte, aficiones y distracciones), y puede ser el resultado de:

- Someter la espalda a un esfuerzo excesivo o irregular.

- Realizar movimientos bruscos mientras el cuerpo se encuentra en una posición inadecuada.

- Déficit de acondicionamiento físico de los músculos de la espalda, del abdomen y del muslo.

- Erosión y desgarros derivados del levantamiento desequilibrado de pesos.

- Larga permanencia en posición de sedestación e inmovilidad.

- Trabajo en una postura de inclinación del cuerpo hacia delante defectuosa o prolongada.

\section{FISIOPATOLOGÍA}

Como se decía anteriormente, la lumbalgia es un síntoma y no una patología como tal, por lo que su fisiopatología dependerá de su etiología, sin embargo, para entender el por qué hay dolor lo explicaremos de una manera resumida: cuando se produce una estimulación nerviosa que el cuerpo detecta como nocivo, las fibras nerviosas van a producir una señal, utilizando glutamato, la cual entrará en la medula espinal por las astas dorsales y viajará hacia el tálamo y posteriormente al encéfalo (4).

La lumbalgia mecánica consiste en el dolor procedente de la irritación mecánica de los receptores de la sensibilidad dolorosa localizados en tercio externo del disco intervertebral, cápsula de articulaciones interapofisarias, ligamentos longitudinales anterior y posterior y estructuras musculoligamentosas de refuerzo de la 
columna lumbosacra. El dolor refleja la alteración de alguna de esas estructuras y produce una limitación de la movilidad que en cierta forma protege a las estructuras dañadas, de que con el movimiento sufran más daño o se perturbe su proceso de reparación (3).

Durante el embarazo: cambios posturales atípicos realizados por la paciente debido al mismo crecimiento y peso del producto. Esto produce un cambio en el centro de gravedad de la persona y esto hace que se aumente la intensidad con la que los músculos lumbares deben de trabajar. También la distención de los músculos abdominales. Como factor hormonal se pueden decir que los estrógenos, progesterona y relaxina reblandecen los cartílagos y ligamentos (7).

Recordemos que la caja abdominal está constituida por:

- Pared posterior: vértebras lumbares, sacro y coxis, articulaciones de los cuerpos vertebrales y las interapofisarias, cartílagos articulares, discos intervertebrales, capsulas articulares y ligamentos, músculos dorsales largos, intermedios y cortos, las fascias toracolumbares.

- Pared anterior: músculos abdominales y aponeurosis de la línea media.

- Paredes laterales: músculos cuadrados de los lomos.

- Techo: musculo diafragma.

- Suelo: suelo pélvico, flexores de la cadera, extensores de la cadera, y abductores de la cadera.

Cabe recalcar que cualquiera de estas estructuras nos puede causar una lumbalgia inespecífica. No es discutible, como se menciona con anterioridad, que las estructuras que contiene esta caja abdominal también nos pueden causar dolor, sin embargo, esto ya sería por definición, una lumbalgia especifica.

\section{CUADRO CLÍNICO}

Cuando el paciente consulta por lumbalgia puede, como lo dice su definición, presentar el dolor lumbar con o sin irradiación a miembros inferiores. También dependiendo de la etiología puede presentar síntomas de radiculopatía, estos serán debilidad, perdida de la sensación, reflejos disminuidos en la raíz nerviosa, pueden coexistir con un dolor radicular.

El dolor radicular es un dolor que se extiende a la pierna sobre su dermatomo y empeora con la tos, estornudo, estiramiento o la prueba del levantamiento de pierna estirada. La causa más frecuente de radiculopatía o dolor radicular es la hernia de disco, sin embargo, esta puede encontrarse en los estudios por imágenes en personas asintomáticas y frecuentemente resuelven por sí solas.

La estenosis espinal vertebral puede causar un dolor al caminar 0 al estar en bipedestación extendida que se irradia hacia uno 0 ambos miembros inferiores $y$ típicamente mejora con el reposo o flexión lumbar (claudicación neurogénica), esto ocurre al haber congestión venosa o isquemia de las raíces nerviosas en la cauda equina por la compresión (1).

Por esta razón, es importante para el medico evaluar al paciente $\mathrm{y}$, como se verá más adelante, descartar las banderas rojas para realizar un diagnóstico preciso.

\section{DIAGNÓSTICO}

El objetivo principal de este capítulo es darle las herramientas al lector para poder realizar un diagnóstico adecuado y con este, mejorar el manejo que se le está dando para que el paciente mejore la actividad, dolor y pronostico y al mismo tiempo se disminuyan los gastos del sector salud en incapacidades, laboratorios o estudios de gabinete innecesarios. Todos los autores estudiados coinciden en que la historia clínica adecuada y un examen físico 
exhaustivo son los pilares para el diagnóstico y manejo de la lumbalgia.

Durante la historia clínica, se debe de preguntar por: ocupación, actividad física, fumado, antecedentes importantes patológicos y cuadros previos.

En este artículo se intentará recomendar al médico una forma, muy conocida, pero a veces obviada, de realizar la historia clínica. Existe una nemotecnia conocida como ALICIA, esta se recomienda por todos los autores sin embargo no con este orden, es algo muy simple para recordar como ordenar la historia clínica, A: tiempo de evolución y como o porque inicio, L: localización, I: intensidad en una escala del 1-10 (siendo el 10 como el dolor más fuerte que ha tenido en la vida), C: características del dolor, I: Irradiación, (¿el dolor es localizado o afecta también otras zonas?), A: alivia o empeora (pueden ser movimientos, actividades, reposo, medicamentos).

Esta nemotecnia es muy útil y la queremos recordar a los médicos tratantes ya que, aun siendo tan conocida y manejada por muchos, lastimosamente algunas veces es obviada en los servicios de emergencias.

En el examen físico se deberá de evaluar IMC, la fuerza, sensibilidad, reflejos de miembros inferiores, también la inspección, palpación y rangos de movimientos de la columna lumbar para evaluar dolor, restricción o espasmos musculares, esto se puede evaluar con la prueba de Schober Modificada (10).

Las pruebas positivas de Lasègue, Bragard y Cruzado sobre Lasègue nos dan un valor predictivo de radiculopatía y hernia de disco (2). Hay que tomar en cuenta que lo que antes se conocía como lumbociatica es un dolor radicular que se extiende desde la zona lumbar hacia miembro inferior por todo el dermatomo y por la parte dorsal, si al realizar la prueba de pierna recta extendida le duele solo la parte posterior del muslo, se considera negativa la prueba y no se deberá de realizar el diagnóstico del mismo.
La prueba de Patrick y Gaenslen pueden ayudarnos a realizar un diagnóstico diferencial entre patología coxofemoral, sacroileaca o lumbar (12).

El adecuado examen físico realiza el descarte de las banderas rojas o bien encuentra alguna de las tantas etiologías de la lumbalgia y con esta información se iniciará el manejo óptimo para cada situación.

Cuando hay dudas o banderas rojas se va a requerir de diferentes estudios de imágenes como radiografías simples Anteroposterior y Lateral, Resonancia magnética y tomografía axial computarizada, también se pueden solicitar electromiografías para valorar el daño neuronal de la extremidad. Los estudios de imágenes, según la totalidad de los autores revisados, deberán de ser solo en casos seleccionados y justificados por las banderas rojas.

Los estudios de laboratorio solo se deberán pedir en caso de que se sospeche de una patología en específico y deberá de ir justificada, ya que no hay estudios de laboratorios que mejoren el pronóstico o ayuden con el manejo de una lumbalgia inespecífica.

\section{Tratamiento}

El tratamiento y manejo de la Lumbalgia dependerá de su etiología, sin embargo, esta revisión bibliográfica se enfocará en la lumbalgia inespecífica.

La lumbalgia inespecífica aguda deberá de tratarse de la mejor manera, según como se explica en este artículo, para evitar su evolución a crónica. Las lumbalgias crónicas son las más complicadas para la vida del paciente y para los costos del servicio de salud.

En la figura 1, se podrá observar como recomendamos manejar al paciente en la atención primaria de la lumbalgia aguda.

Debemos de evaluar al paciente y descartar todo signo de alarma que se expuso 
anteriormente, una vez realizado ese proceso se debe de explicar al paciente que su cuadro es benigno y que no requiere de incapacidad.

En el sector de trabajadores de campo, por experiencia de los autores de este artículo, se evidencia como la población está acostumbrada a pedir incapacidad por lumbalgia, esto no es incorrecto en caso de lumbalgia específica, sin embargo, en el caso de la lumbalgia inespecífica, debería de tratarse preventivamente, conservador $y$ médico.

Tratamiento preventivo y terapéuticas: La educación al paciente es muy importante, higiene postural, actividad física, evitar reposo en cama, evitar sedentarismo, disminución de peso o bien evitar la ganancia de peso, ejercicios de estiramiento y fortalecimiento, mejorar condición física, fisioterapia, yoga, taichí o Pilates, electroterapia, etc. nunca reposo en cama ya que prolonga la discapacidad (7), aeróbicos acuáticos y acupuntura. Muchas veces el paciente no entiende el por qué se le envía sin incapacidad a realizar más ejercicio del que ya hace en el trabajo. Sin embargo, ahí tiene que intervenir el médico y explicar la fisiopatología de la debilidad muscular, contracciones musculares e inflamación de las diferentes estructuras y como estas van a mejorar con el fortalecimiento y utilización de estos.

Se ha evidenciado que el otorgar incapacidad a los pacientes con lumbalgia solo empeora el cuadro, ya que el paciente no toma estos días para su rehabilitación, si no para descansar. Por esta misma razón se deben de continuar las labores por parte del paciente y realizar actividad física y no otorgar la incapacidad que tanto pide este. Muchas veces el paciente lo único que desea es saber la causa de su dolor y desea que este se detenga ya que interfiere con su calidad de vida tanto social como laboral. El paciente desarrolla pensamientos de inutilidad, por lo que el medico debe de dar apoyo emocional para que el paciente no presente banderas amarillas y tenga menos riesgos de desarrollar cronificación del cuadro.

Tratamiento conservador: aun en la práctica se logra observar como los médicos tratantes manejan el paciente con lumbalgia como si todos tuvieran banderas rojas, y someten a los pacientes a estudios de imágenes, valoraciones por especialistas y hasta referencias para infiltraciones $y$ cirugías. Debemos que recordar que los estudios de imágenes solo deben realizarse ante la presencia de banderas rojas y debería de estar justificado con el hallazgo en el examen físico.

Las valoraciones por especialistas deberían realizarse únicamente ante el hallazgo de las banderas rojas y estudios de imágenes pertinentes reportadas por un Radiólogo asegurando y confirmando el diagnóstico. Las infiltraciones y cirugías según la bibliografía revisada son decisiones que tomaran solo los especialistas, y son para un número muy seleccionado de pacientes, por lo que el médico tratante de primer nivel solo deberá de preocuparse por descartar banderas rojas.

Tratamiento médico: hay discrepancias, entre los artículos revisados, en cuanto al tratamiento médico que debería de indicarse en la lumbalgia inespecífica. Los medicamentos indicados para lumbalgia son, según lo leído: Acetaminofén, AINES, relajantes musculares, antidepresivos y opioides.

Se recomienda según artículos como primera línea AINES (5), se pueden utilizar COX-2 si no tolera los AINES convencionales. Se utilizará acetaminofén como segunda línea, no obstante, otros estudios lo indican de manera inversa (4). Los relajantes musculares los recomiendan por un periodo corto menor a 2 semanas sin embargo tienen muchos efectos secundarios y pueden causar dependencia por lo que se recomienda no utilizarlos, de igual manera 
no los encontramos en la CCSS en atención primaria.

Los opioides, como el Tramadol, están indicados cuando los AINES no logran el efecto deseado.

Los antidepresivos pueden ser usados para aumentar la serotonina y norepinefrina para aliviar el dolor (12). Pese a que otros autores descartan a los antidepresivos como tratamiento para la lumbalgia (4).

Se desea recalcar que la mejor evolución de las lumbalgias en la totalidad de los estudios revisados fueron los ejercicios enfocados en estiramiento y fortalecimiento de musculatura lumbar y abdominal, actividad física, higiene postural, actividades como yoga, Pilates e hidroterapia. Los medicamentos tomados por el paciente son coadyuvantes para la tolerancia de la rehabilitación.

Luego de 2 semanas de apego al tratamiento y mejoría del dolor, se debe de alentar al paciente a aumentar el número de veces y ejercicios que realiza a la semana. Esto para aumentar su condición física y fuerza muscular, a la vez que se deberá de disminuir la dosis del medicamento.

Si en 4 semanas no ha mejorado el dolor y el paciente asegura el apego al tratamiento terapéutico y médico, entonces hay que indagar por banderas amarillas, estas pueden estar evitando que el paciente mejore, por lo que va a requerir de referencia para terapia cognitivo conductual. Esta se ha evidenciado en numerosos estudios que mejora significativamente el dolor del paciente con banderas amarillas presentes.

Si han transcurrido 6 semanas no mejoran los síntomas, se convierte en una bandera roja por lo que va a requerir de estudios de imágenes y una nueva valoración de manera más exhaustiva (8).

Se realiza un esquema para ayudar al lector a recordar el orden secuencial del manejo de lumbalgia.

\section{COMPLICACIONES}

La mayor complicación de la lumbalgia inespecífica aguda es que se cronifique. Sin embargo, esto es solo hasta haber descartado todas las banderas rojas.

Cuando la lumbalgia es una condición crónica la cual en la mayoría de los casos es fluctuante y presenta episodios de leve a moderado en intensidad del dolor, la limitación dependerá de la evolución del dolor. Patologías como asma, cefalea, diabetes o pobre salud mental (ansiedad, depresión o TAG), pueden contribuir a una lumbalgia en comparación con las personas con una buena salud.

Continuar con trabajos pesados, posturas inadecuadas, cansancio y distracciones durante el trabajo pueden contribuir con episodios de lumbalgia (1).

Las otras complicaciones más severas serán a nivel de la calidad de vida del paciente, ya que esto puede causarle al paciente un aislamiento importante tanto dentro como fuera del trabajo, el paciente no podrá realizar las actividades previas y no podrá tener un crecimiento profesional, ni adquirir la misma riqueza que sus colegas de trabajo (1).

A nivel de los gastos en salud, se ha demostrado que el $25 \%$ de los consultantes de atención primaria por lumbalgia reciben una radiografía sin necesitarla y/o no justificada. El $16 \%$ una tomografía axial computarizada no justificada, y hasta el $88 \%$ tratamiento médico inútil ante la lumbalgia (9). Esto es importante ya que con un tema tan consultado y frecuente como lo es la lumbalgia se nota como los gastos aumentan cada vez más utilizando recursos que no son requeridos para su manejo ni evolución (14). Los gastos aumentan aún más cuando son trasladados en ambulancias o admitidos al hospital sin requerirlo (18). 
Figura 1. Esquema del manejo de lumbalgia aguda

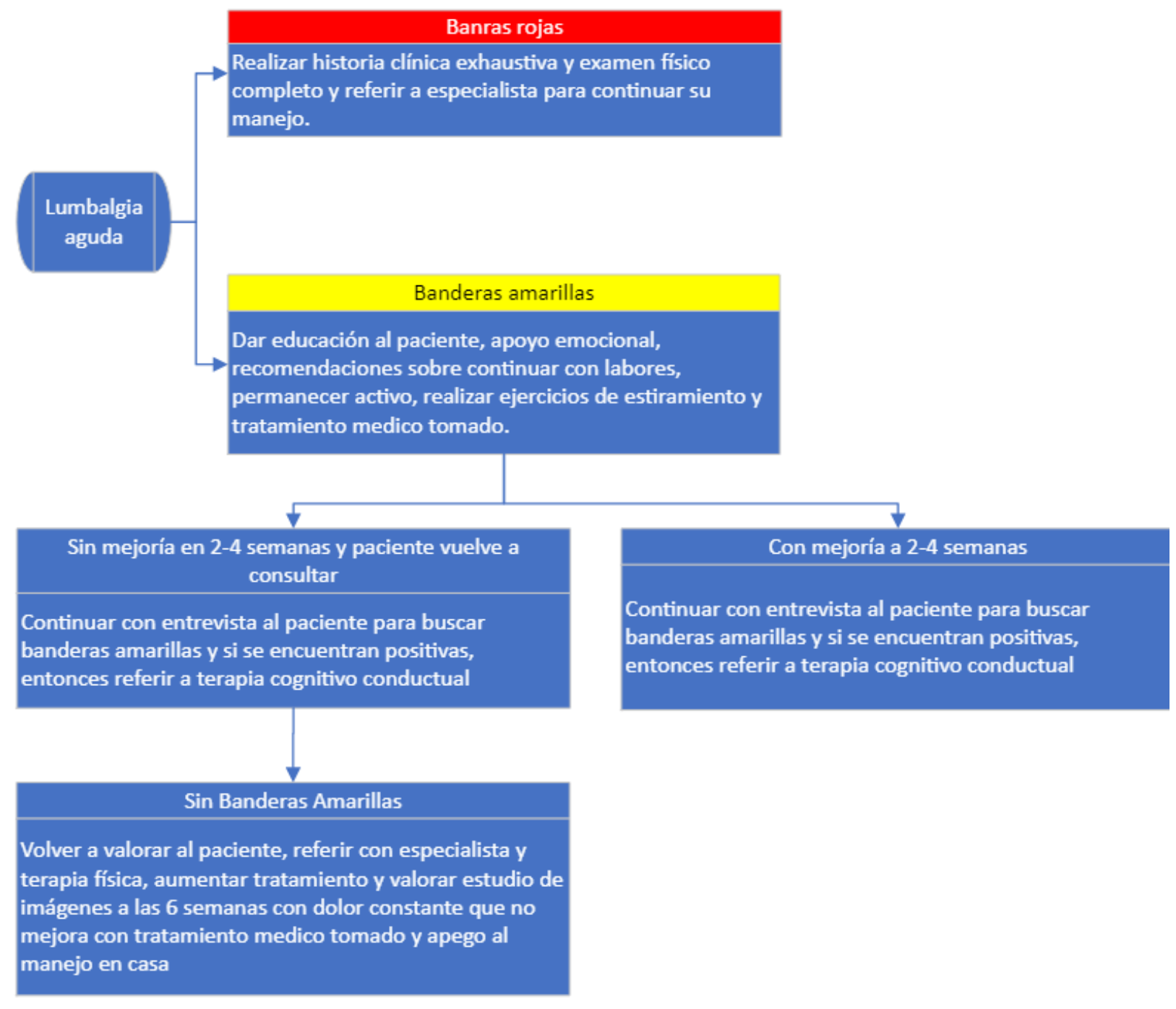

Fuente. Propia de los autores

\section{PRONÓSTICO}

El factor pronóstico de mayor importancia para la lumbalgia será la presencia de factores de riesgo y las banderas amarillas. Cuando hay uno o más factores presentes, es muy probable que el paciente presente lumbalgia crónica y requiera de numerosas visitas y tratamiento médicos y terapéutico por más tiempo. También va a requerir de un manejo multidisciplinario lo que hará que el costo para su mejoría sea aún mayor.
Muchos pacientes que refieren lumbalgia también asocian dolores en otras partes del cuerpo y presentan problemas físicos y mentales, comparado con pacientes sin lumbalgia (1).

Personas con dolor van a presentar cambios de humor, problemas para socializar, incapacidad para ser productivos y activos (5).

Buenos comportamientos: actividad física, dieta, ejercicios de rehabilitación, no abandono del manejo impuesto por el médico, continuar con sus labores, control 
del estrés, ponerse metas y cumplirlas y comunicarle a familiares $y$ amigos del proceso para que se sienta acompañado.

Malos comportamientos: frustración por dolor, quejarse del dolor, ansiedad, depresión, sedentarismo, no toma de medicamento, no realizar ejercicios de rehabilitación, no disminuir de peso y abuso de sustancias, miedo al dolor y no realizar actividad física (4).

\section{CONCLUSIONES}

Como se logra evidenciar, los numerosos autores estudiados concuerdan en mejorar de manera uniforme el manejo que se le da a las lumbalgias en la atención primaria.

En la práctica clínica, según la experiencia de ambos médicos autores de este artículo, se logran encontrar diferentes manejos que no están descritos en la literatura, como por ejemplo: reposo absoluto, ausencia por incapacidad por lumbalgias mecánicas de hasta 7 días, antidepresivos, estudios de imágenes, referencias a especialistas $y$ traslados a hospitales. El paciente se ha acostumbrado, por un mal manejo, a un reposo absoluto en cama y además a poder conseguir ausencias laborales por incapacidad por lumbalgias, tanto así que cuando se les maneja de la forma adecuada, estos pacientes presentan enojo e insatisfacción por la manera en que son abordados.

En muchas ocasiones, el médico quiere conseguir un diagnóstico especifico y darle al paciente una explicación de su dolor; no obstante, en el caso de lumbalgia la explicación más frecuente es la causa inespecífica y el manejo es conservador con ejercicios en el hogar, sin suspender labores y sin realizar reposo absoluto.

Con este artículo se espera que el manejo, al menos en Costa Rica, se unifique y que los estudios de imágenes, laboratorios, incapacidades, traslados a hospitales $y$ referencias a especialistas sean justificados y así poder liberar los servicios de salud, de pacientes innecesarios que no requieren de mayor atención.

\section{Los autores declaran no tener conflicto de interés.}

\section{REFERENCIAS}

1. Hartvigsen J, Hancock MJ, Kongsted A, Louw Q, Ferreira ML, Genevay S, et al. What low back pain is and why we need to pay attention. Lancet. 2018;391(10137):2356-67.

2. Will JS, Bury DC, Miller JA. Mechanical low back pain. Am Fam Physician. 2018;98(7):421-8.

3. Sánchez-Pinilla RO. ¿Cuáles son los ejercicios indicados en la lumbalgia crónica y en la hernia discal? FMC - Form Médica Contin Aten Primaria. 2020;27(1):34-46.

4. Alleva J, Hudgins T, Belous J, Kristin Origenes A. Chronic low back pain. Dis Mon. 2016;62(9):3303.

5. Casser H-R, Seddigh S, Rauschmann M. Acute lumbar back pain: Investigation, differential diagnosis, and treatment. Dtsch Arztebl Int [Internet]. 2016; Available from: http://dx.doi.org/10.3238/arztebl.2016.0223

6. Selkirk SM, Ruff R. Low back pain, radiculopathy Handb Clin Neurol. 2016;136:1027-33.

7. Gallo-Padilla D, Gallo-Padilla C, Gallo-Vallejo FJ, Gallo-Vallejo JL. Low back pain during pregnancy. Multidisciplinary approach. Semergen. 2016;42(6):e59-64.

8. Pérez Delgado FJ, Gil Sánchez C, Izquierdo Gomar AB. Lumbalgia resistente a tratamiento. Med gen fam. 2016;5(2):68-70.

9. Norberg M, Norberg M-A. Lumbalgias comunes: discapacidad y técnicas de evaluación, incidencias socioeconómicas. EMC - Apar Locomot. 2016;49(4):1-7.

10. Movasat Hajkhan A, Bohórquez Heras C, Turrión Nieves A, Álvarez de Mon Soto M. Protocolo diagnóstico del dolor lumbar mecánico. Medicine. 2017;12(26):1541-5.

11. BaRutell $\mathrm{C}$, doMlngo $\mathrm{F}$, RodRíguez $R$. Lumbalgias: técnicas de diagnóstico. Dolor® Investigación, Clínica \& Terapéutica. 2018;33:138-141.

12. Urits I, Burshtein A, Sharma M, Testa L, Gold PA, Orhurhu $\mathrm{V}$, et al. Low back pain, a comprehensive review: Pathophysiology, diagnosis, and treatment. Curr Pain Headache Rep. 2019;23(3):23

13. Maher C, Underwood M, Buchbinder R. Nonspecific low back pain. Lancet. 2017;389(10070):736-47. 
14. Carregaro RL. Management of non-serious low back pain in the context of emergency care. Is it worth the cost? The Lancet Regional Health Western Pacific. 2021;7(100105):100105.

15. Violante FS, Mattioli S, Bonfiglioli R. Low-back pain. Handb Clin Neurol. 2015;131:397-410.

16. Patrick N, Emanski E, Knaub MA. Acute and chronic low back pain. Med Clin North Am. 2016;100(1):169-81.

17. Petersen $T$, Laslett $M$, Juhl C. Clinical classification in low back pain: best-evidence diagnostic rules based on systematic reviews. BMC Musculoskelet Disord [Internet]. 2017;18(1). Available from: http://dx.doi.org/10.1186/s12891017-1549-6

18. Coombs DM, Machado GC, Richards B, Wilson R, Chan J, Storey $\mathrm{H}$, et al. Healthcare costs due to low back pain in the emergency department and inpatient setting in Sydney, Australia. The Lancet Regional Health - Western Pacific. 2021;7(100089):100089. 